\title{
National Meeting of the Spanish Society of Nitrogen Fixation (XV SEFIN)
}

\author{
Fernando González-Andrés ${ }^{1}$ • Euan James ${ }^{2}$
}

Received: 10 December 2015 / Accepted: 14 December 2015 / Published online: 5 January 2016

(C) Springer Science+Business Media Dordrecht 2016

This special issue of Symbiosis contains a selection of the papers presented at the XV SEFIN, the National Meeting of the Spanish Society of Nitrogen Fixation (SEFIN in the Spanish acronym), held in León (Spain) from 16th to 18th June 2015. Since 2008, the SEFIN meeting has been celebrated with the PortugueseSpanish Congress on Nitrogen Fixation, and this was the 4 th occasion. The slogan of this international event was "The bridge between biotechnology, agriculture and environment", which expresses the intention and efforts of the SEFIN to transfer scientific findings and knowledge to a broad spectrum of the scientific society including the biotechnological industry, agriculture and those involved in the protection of the environment. The Congress was an international event, which brought together more than 110 scientists from four continents: Europe, Asia (Middle East), America (Latin America and the Caribbean) and Africa.

The papers contained in this special issue cover all aspects of the biological nitrogen fixation and beneficial plant-microbe interactions: i) four papers on Ecology, diversity, and evolution of Plant Probiotic Microorganisms (PPM); (ii) three papers on genetics, genomics and proteomics of PPM and their associated plants; iii) one paper about plant-microorganism interactions; iv) two papers on the physiology and biochemistry of beneficial microorganisms and associated plants; v) and three papers on inoculants for agriculture and environmental sci-

Fernando González-Andrés

fgona@unileon.es

Universidad de León, León, Spain

2 The James Hutton Institute, Invergowrie, Dundee, UK ence. Thus, the topics cover aspects from the molecular level and the physiological one to field trials, and from regulatory aspects to the communication between plants and microorganisms.

The Congress consisted of keynote lectures that were given by Prof. Yaakov Okon (Department of Plant Pathology and Microbiology, Faculty of Agriculture, Food and Environment, The Hebrew University of Jerusalem, Israel) and Dr. Euan James (The James Hutton Institute, Invergowrie, Dundee, UK), linked with the five sessions mentioned above. The sessions consisted of 36 contributed oral presentations, plus 59 posters, all were authored by young graduate students in association with their major professors and the lab teams from their institutions.

The ancient and historical city of León in the interior northwest Spain provided a wonderful backdrop to the Congress. León is a beautiful, quiet city, enjoyable to walk around with 139,000 inhabitants. It is a small city with a long history, and a lot of culture and traditions. A field trip was organized to the World Heritage site of

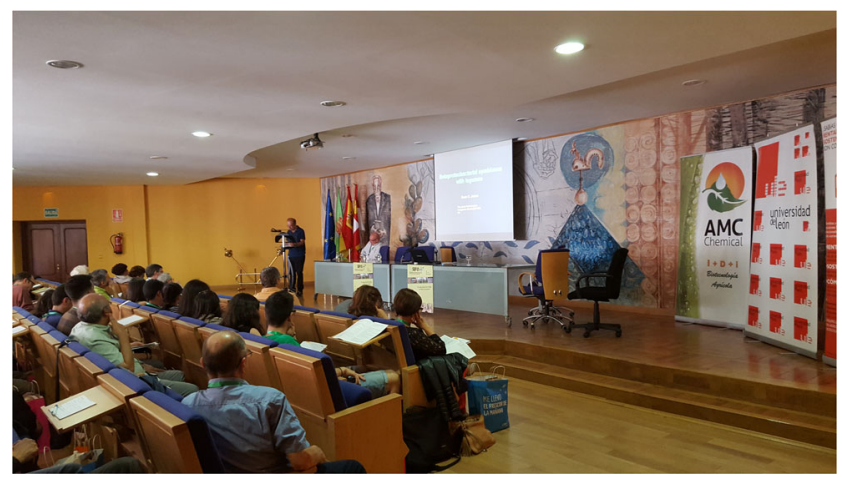

Fig. 1 Introduction by one of the keynote speakers during the opening ceremony of the XV SEFIN Congress 


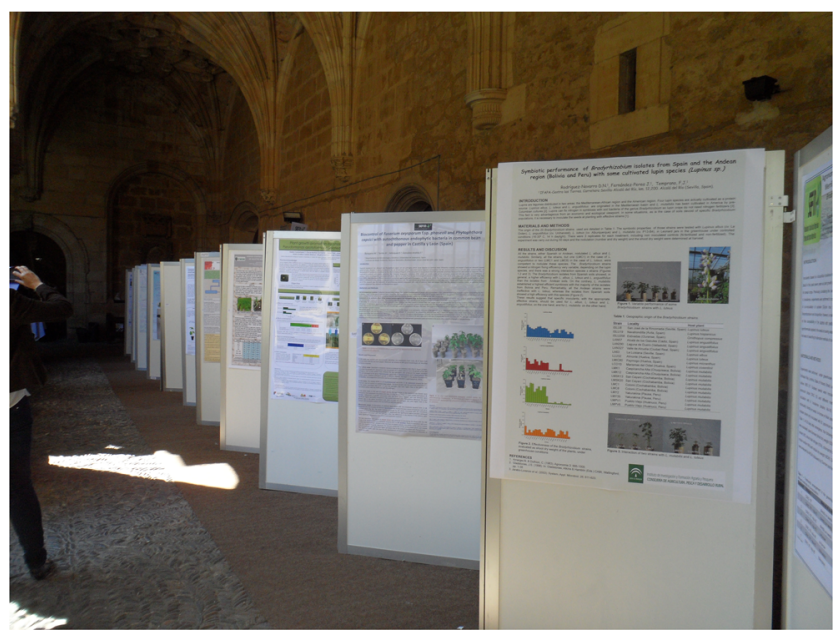

Fig. 2 A view of some of the contributed posters displayed in the cloister of the national monument "San Marcos" in the city of León (Spain), the venue of the Congress

Las Médulas in the county of Bierzo, in the northwestern part of León province. The organizers of the conference led by Dr. Fernando González-Andrés from University of León (as the President of the Organizing Committee) and Dr. Eustoquio Martínez-Molina from

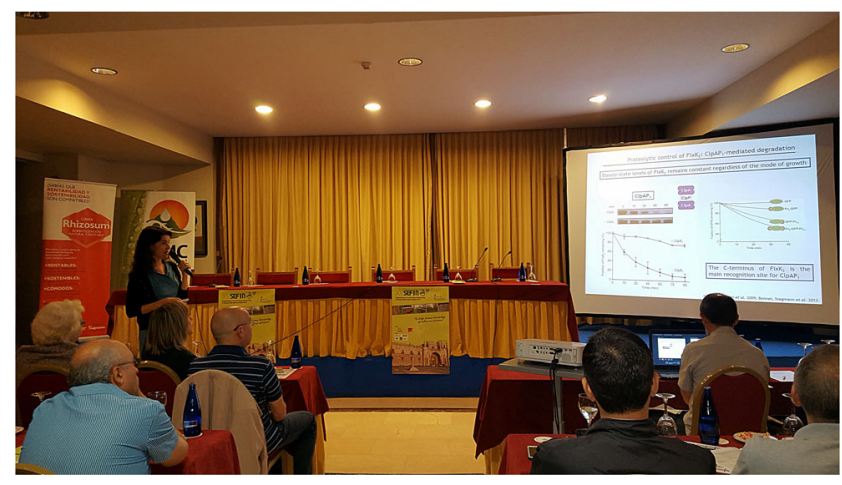

Fig. 3 An oral presentation in the contributed papers session

the University of Salamanca (as the President of SEFIN) are grateful to the co-organizers of the event and acknowledge the University of León and the Spanish Society of Nitrogen Fixation for their financial support. The staff of both institutions were involved in the organization and are thanked. The organizers are also grateful to the sponsors of the Congress including the enterprises Agrocode Bioscience, Agrogenia, AMC Chemical, Biomasa Peninsular, BYDT, and Laboratorios Microkit, for their support (Figs. 1, 2, and 3). 